http://jmscr.igmpublication.org/home/ ISSN (e)-2347-176x ISSN (p) 2455-0450 crossref DOI: https://dx.doi.org/10.18535/jmscr/v7i8.162

Journal Of Medical Science And Clinical Research

\title{
The Rare 'Gliosarcoma': Experience at Tertiary Care Centre
}

\author{
Authors \\ Vijay Ramita ${ }^{1 *}$, Mathur Kusum ${ }^{2}$ \\ ${ }^{1}$ Postgraduate Student, ${ }^{2}$ Senior Professor \\ Department of Pathology, SMS Medical College, Jaipur, Rajasthan, India \\ Corresponding Author \\ Vijay Ramita
}

\begin{abstract}
Introduction: Gliosarcoma is rare primary malignant tumor of brain and is a histological variant of glioblastoma. This supratentorial tumor usually affects older age groups and is extremely rare in paediatric population. Morphologically characterized by a biphasic pattern having a gliomatous GFAP positive component alternating with a vimentin positive sarcomatous component. Immunohistochemical analysis is of immense help in confirmatory diagnosis by pathologists. Being a WHO grade IV tumor it bears a poor prognosis despite concomitant surgical and chemo radiation therapies. The present study was conducted to study the clinical and histomorphological features of gliosarcoma at a tertiary care institute.

Materials and Methods: A retrospective cross-sectional study was conducted at SMS Medical College, Jaipur during study period January 2013 to December 2018.

Results: 12 cases of gliosarcoma were reviewed out of which 4 (33.3\%) cases belonged to paediatric age group. Mean age of study subjects was 32.9 years. Most frequent site of tumor was left temporoparietal (25\%) followed by left frontotemporoparietal (16.7\%). All patients presented with headache and vomiting. The most frequent associated symptom was altered consciousness followed by seizures. Light microscopic studies revealed mixed tumor with glial and sarcomatous components showing immunopositivity for GFAP and Vimentin respectively and a high proliferative index.

Conclusion: In present study a younger population was affected, so gliosarcoma should be kept as a differential diagnosis while evaluating supratentorial tumor in pediatric population as well. Immunohistochemical studies must be carried out to reach at the accurate diagnosis and to support treatment strategies.

Keywords: Glial tumor, gliosarcoma, immunohistochemistry, paediatric.
\end{abstract}

\section{Introduction}

Gliosarcoma is a rare primary malignant tumor of central nervous system and is considered a variant of glioblastoma multiforme (GBM) representing only $2 \%$ of all GBM cases. ${ }^{1}$ It usually occurs in elderly between fifth and sixth decade of life (average age- 54 years) and has slight male preponderance (M:F-2:1). ${ }^{2}$ Primary gliosarcoma preferentially locate supratentorially in temporal lobes. Histomorphologically, it has biphasic pattern with both glial and malignant mesenchymal components. These are highly aggressive malignant gliomas with WHO grade IV. ${ }^{3}$ Mainstay of therapy is surgery followed by adjuvant radiotherapy and temozolomide-based 
chemotherapy, however, this tumor has still poor prognosis. ${ }^{4}$

The present study has been conducted with the aim to study the clinical, radiological and histopathological features of gliosarcoma at a tertiary care institute.

\section{Materials and Methods}

A retrospective cross sectional study was conducted at SMS Medical College, Jaipur. Paraffin blocks of cases reported as gliosarcoma during the time period from January 2013 to December 2018 were retrieved. Age, sex, clinical history and imaging features of each case were registered from the case records. Blocks were sectioned and stained with Hematoxylin and Eosin, special stains and immunohistochemistry were applied and were examined under light microscope.

\section{Results}

During the study period a total of 12 cases of gliosarcoma were obtained. There was a wide age range from 12 to 70 years with the mean age being 32.9 years.

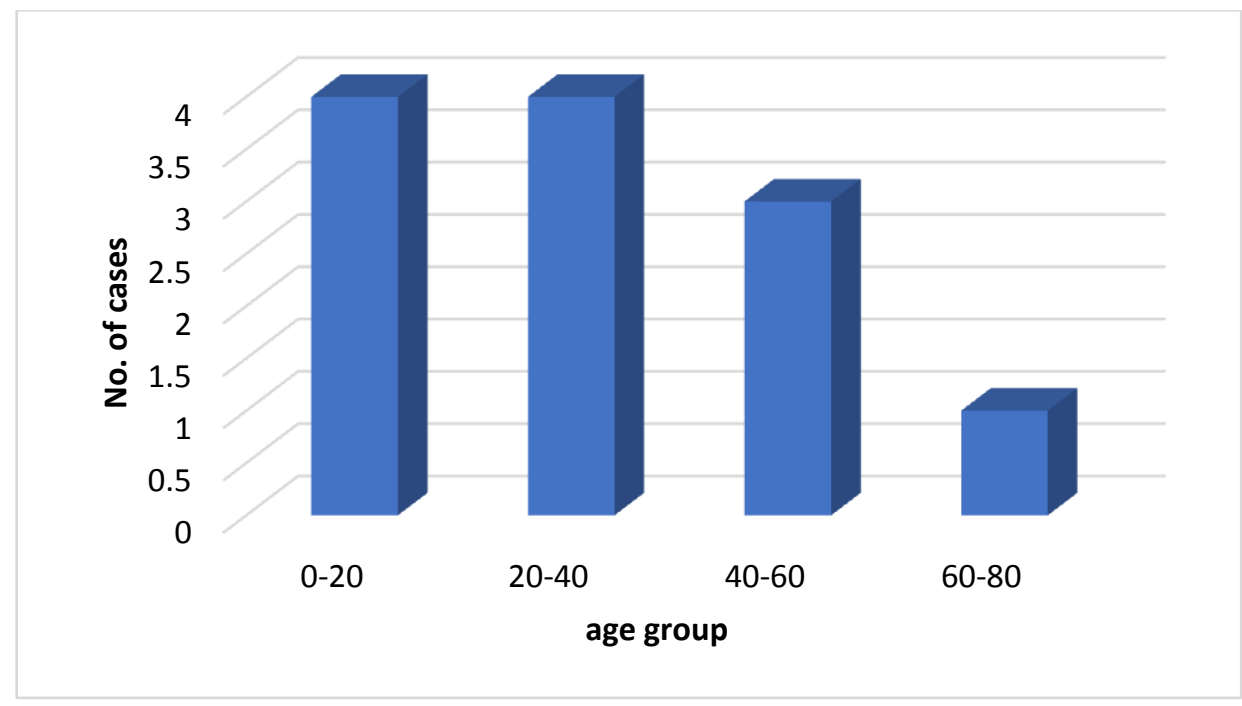

Figure 1: Age distribution of cases

Four cases $(33.3 \%)$ belonged to pediatric age group and 8 cases $(66.7 \%)$ occured in adults.
Males constituted 8 cases $(66.7 \%)$ and females constituted 4 cases $(33.3 \%)$.

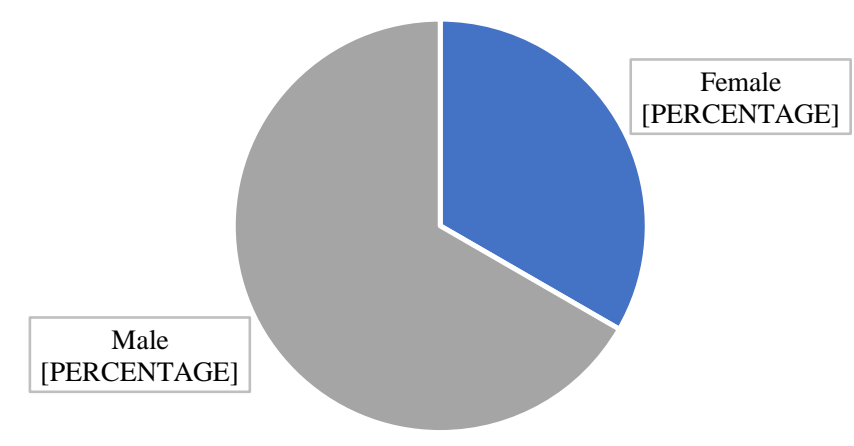

Figure 2: Sex distribution of cases 
Clinically, all cases presented with headache associated with vomiting. 8 cases $(66.7 \%)$ had a history of either giddiness, confusion or loss of consciousness. 6 cases $(50 \%)$ presented with a history of seizures. 5 cases $(41.7 \%)$ revealed history of neurocognitive decline and 5 cases $(41.7 \%)$ had weakness in extremities.

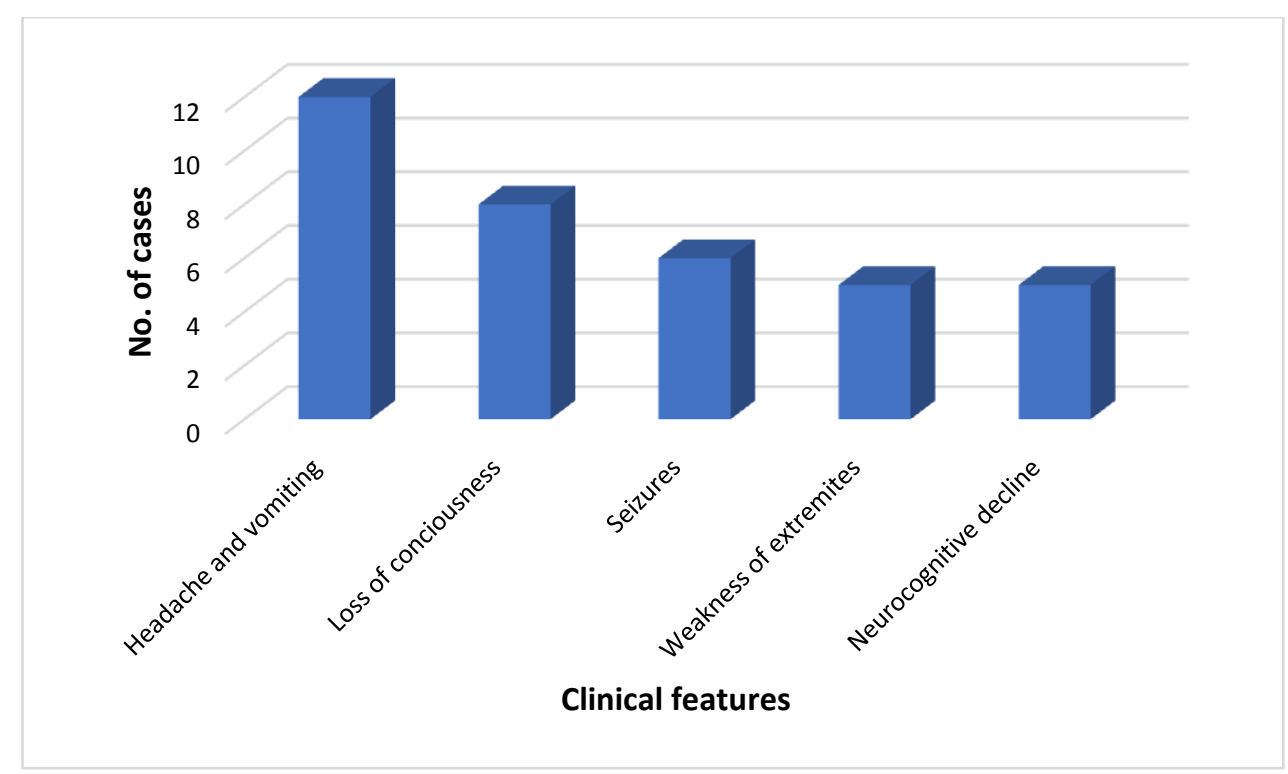

Figure 3: Clinical Features

The most frequent tumor location in our study was Left Temporoparietal lobe observed in $25 \%$ (3/12) cases followed by Left frontotemporoparietal lobe seen in $16.7 \%(2 / 12)$ cases.

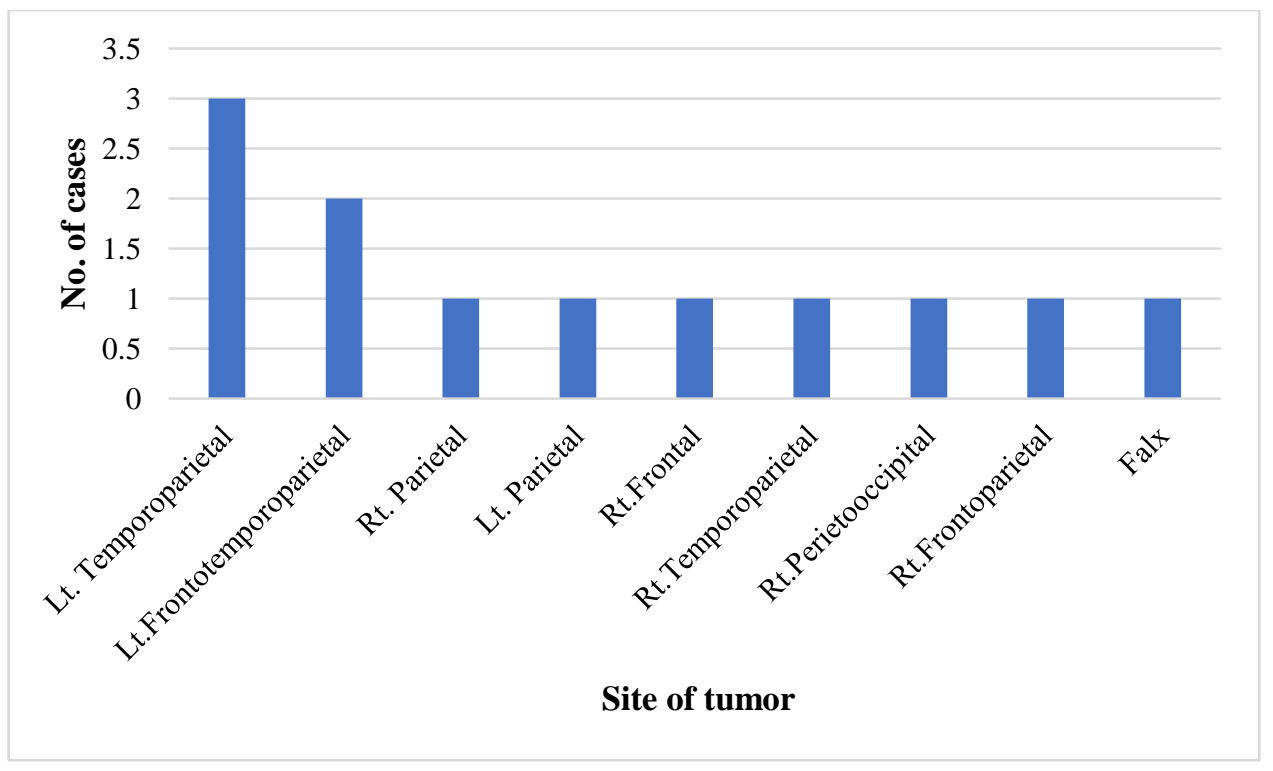

Figure 4: Distribution of tumor location

Radiologically and histopathologically all cases in our study, revealed similar features. Computed tomography scan of brain revealed a mildly enhancing heterogenous intra-axial spaceoccupying lesion with perilesional edema and mass effect suggestive of an aggressive lesion, mainly glioma. Magnetic resonance imaging also showed similar findings. All tumors underwent surgical excision and were sent for microscopic evaluation. 

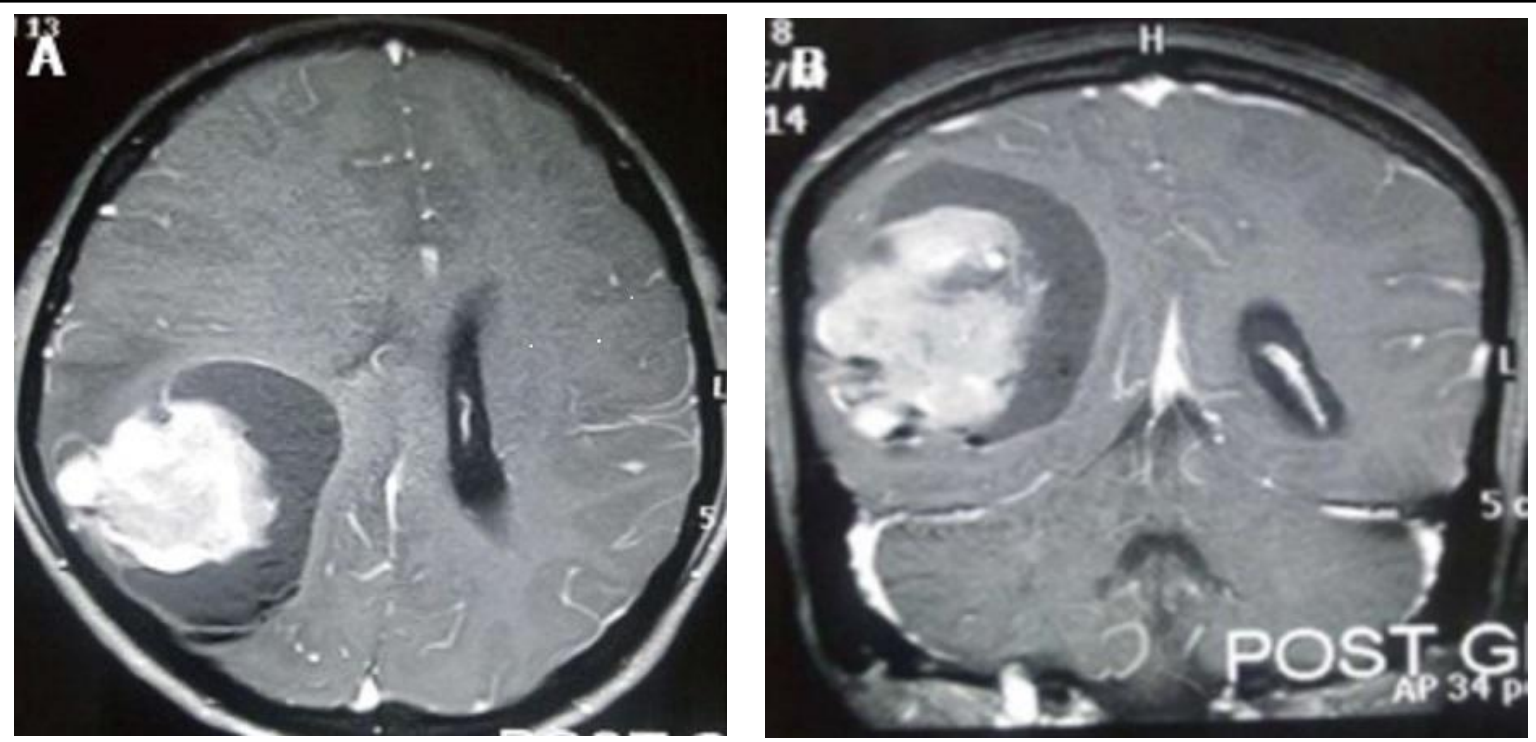

Figure 5: CT scan brain (axial and coronal) Showing heterogeneous mass lesion in Rt. Parietooccipital lobe with perilesional edema.

Histopathological specimen were examined under light microscope after routine processing and staining. The tumor showed a biphasic tissue pattern comprising of alternating areas displaying glial and mesenchymal differentiation. The glial component appeared to be astrocytic and showed cellular areas of glial cells with pleomorphic, vesicular nuclei and prominent nucleoli admixed with mitosis, increased vascularity, and pericytic hyperplasia. These are the typical features of a glioblastoma. The sarcomatous component consists of densely packed spindle cells having pleomorphic, hyperchromatic nuclei and scanty cytoplasm arranged in fascicles and in perithelial arrangement. Areas of necrosis and haemorrhage were observed. On applying Reticulin stain, individual tumor cell was seen to be surrounded by reticulin fibres suggesting the presence of sarcomatous component.

On immunohistochemical analysis tumor cells of glial component were positive for glial fibrillary acidic protein (GFAP) and that of mesenchymal component were reactive to vimentin. Cells show no immunoreactivity for desmin, smooth muscle actin, EMA, CK20, CK7 and CD34. IHC confirmed the biphasic nature of the tumor. The tumor cells showed high proliferative (ki-67) index.
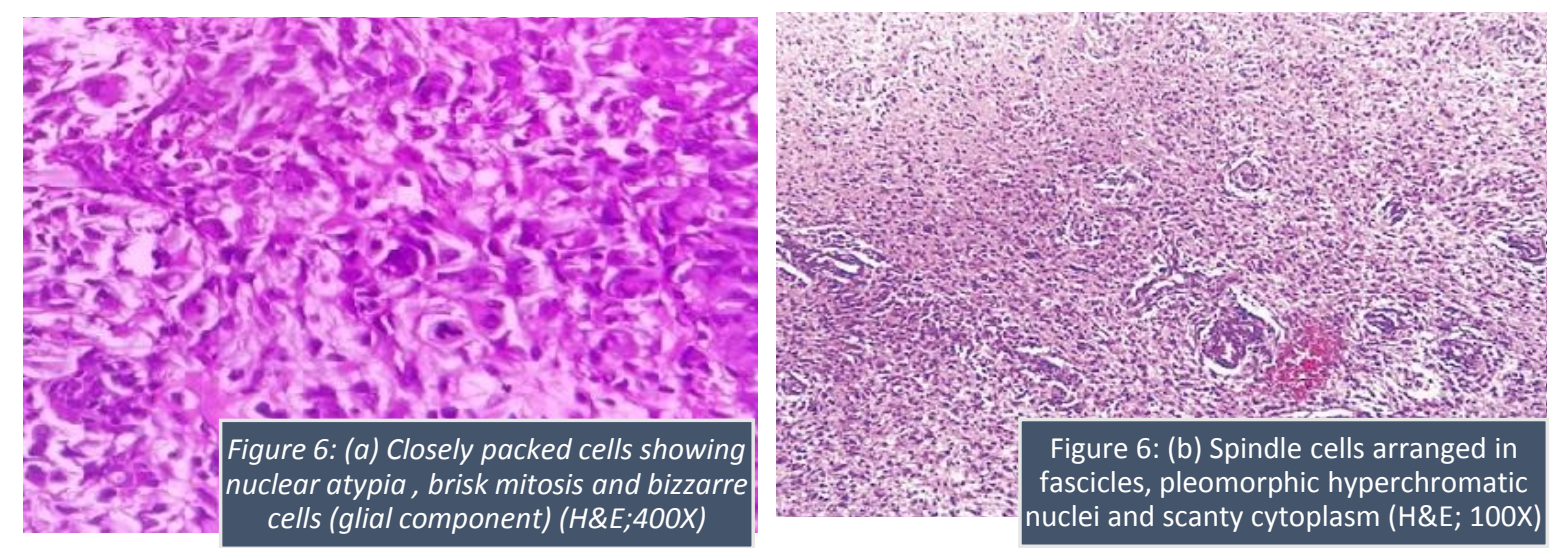

Figure 6 : Light microscopic findings 


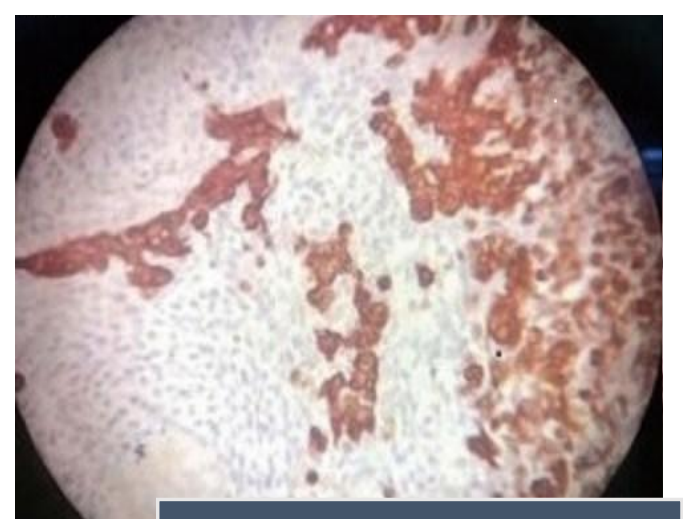

Glial cells show GFAP

immunopositivity (GFAP, 400X)

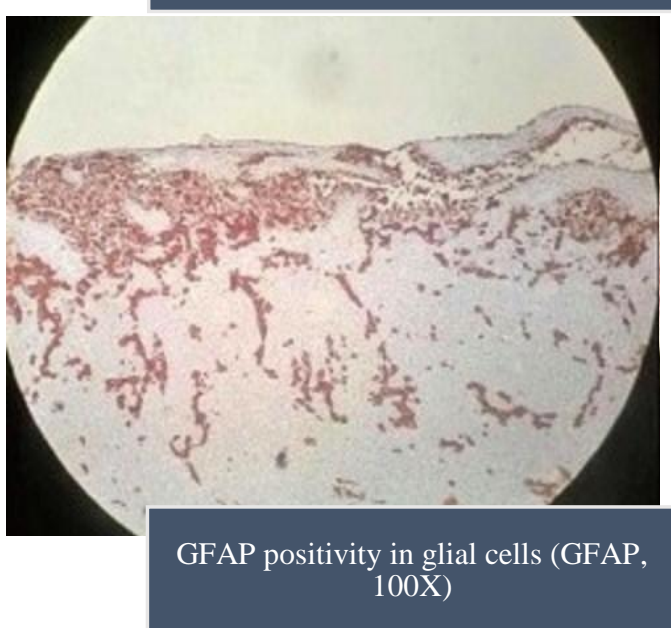

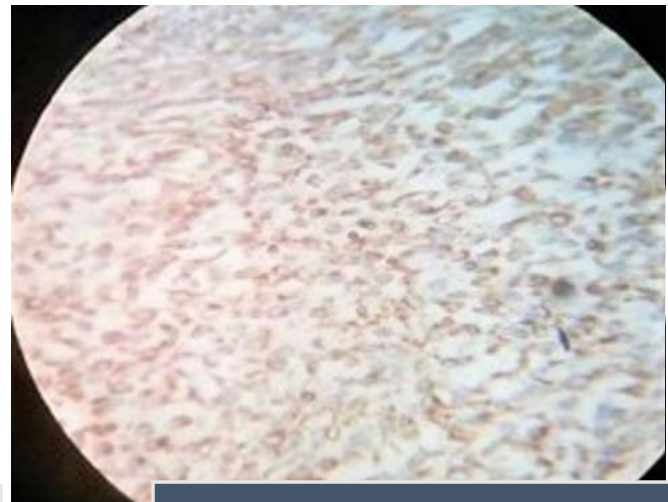

Spindle cells positive for vimentin (Vimentin, 400X)

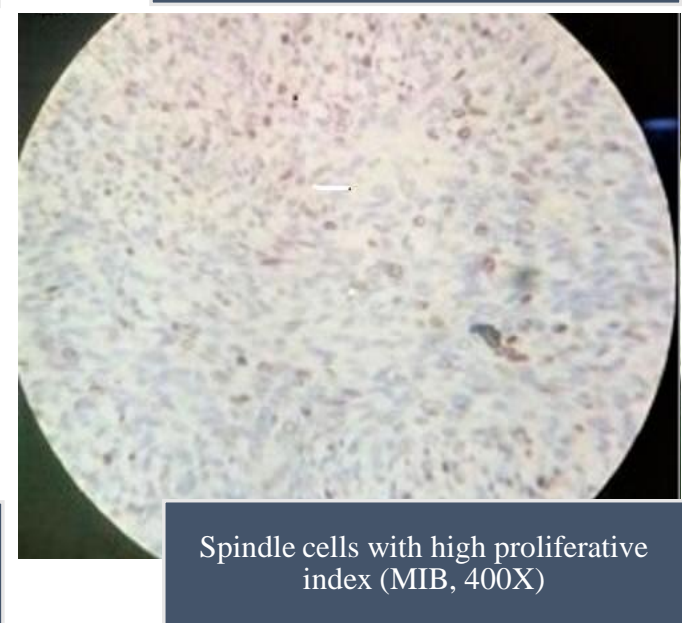

Figure 7: Immunohistochemical profile of gliosarcoma

\section{Discussion}

Gliosarcoma was first described by Stroebe in 1895. Gliosarcoma is an exceptionally rare malignancy of brain that has been categorized by WHO, as a variant of glioblastoma and assigned grade IV. ${ }^{3}$ These tumors usually present in the age of $50-60$ years. $^{2}$ It is extremely rare in pediatric age group. In our study 4 cases were of pediatric age and the mean age of presentation was 32.9 years. The younger age of distribution was also seen in other series. ${ }^{6,7,8}$

Clinically, most of the cases present with symptoms of increased intracranial pressure and expanding intracranial mass such as headache, blurring of vision, hemiparesis, seizures and cognitive decline. ${ }^{9}$

Gliosarcomas locate in supratentorial region, most frequently in temporal region followed by the frontal, parietal and occipital lobes. ${ }^{10}$ In a series by Kumar et al, $37.5 \%$ of patients had primary gliosarcoma located in temporal lobe. ${ }^{7}$ In our study, 4 cases had tumor in Temporoparietal lobe and 2 had tumor located to frontotemporoparietal lobe..

Histologically, gliosarcoma is a mixed tumor composed of both gliomatous and sarcomatous elements. The glial component is histologically similar to typical glioblastoma tissue and shares similar clinical and genetic features. ${ }^{11}$ Exact pathogenesis of gliosarcoma remains unknown, but earlier it was postulated that the sarcomatous components originated from malignant transformation of characteristic hyperplastic blood vessels found in high grade gliomas. ${ }^{12}$ Many studies have now revealed identical genetic alterations such as chromosomal aberrations and cytogenetic imbalances and identical p53 mutations in thesarcomatous and gliomatous elements of gliosarcoma, indicating their monoclonal origin. ${ }^{13}$

The sarcomatous component shows signs of malignant transformation and often demonstrates 
the typical pattern of fibrosarcoma. The high content of connective tissue contributes to the gross appearance of a firm, well circumscribed mass that is often mistaken for meningioma, if attached to duramater. Some cases may show additional lines of mesenchymal differentiations, e.g. cartilage, bone, smooth and striated muscle and even lipomatous tissue. The connective tissue fibers are showed by reticulin and vimentin stains, while GFAP is expressed in the glial part. The demonstration of a clearly malignant mesenchymal GFAP-negative component is essential to distinguish true gliosarcoma from glioblastoma with desmoplasia. Gliosarcomas harbour PTEN mutations, p16INK4-alpha deletions and TP53 mutations just like primary glioblastoma but show infrequent EFGR amplifications. $^{14}$

The gliosarcomas metastasize frequently to the lungs, pleura, lymph nodes, bone marrow, liver, spinal cord, kidney and peripancreatic areas. However, no systemic metastasis was seen in this study. ${ }^{15}$

Therapeutic modalities for gliosarcoma are similar to GBM and tumor resection, postoperative radiation therapy, and chemotherapy are the main treatment modalities. ${ }^{16}$ Treatment with concurrent chemo radiation with temozolomide improved overall survival. ${ }^{17}$ But the prognosis still remains in dismay with the mean survival being 13 months. ${ }^{18}$

The current literature provides a number of distinguishing clinical and pathogenetic features that differentiate gliosarcomas from glioblastomas. These include the predilection for the temporal lobe, potential to appear similar to a meningioma grossly on operation, their increased metastatic potential, and the infrequency of epidermal growth factor receptor (EGFR) mutation in gliosarcomas.

\section{Conclusion}

Gliosarcoma is a rare central nervous system malignancy that carries poor prognosis. It is highly aggressive and although rare in paediatric age group, should be kept in differential diagnosis. Immunohistochemistry has a confirmatory role in diagnosis. The studies focusing on cellular and molecular biology of gliosarcoma are requiredto improve treatment modalities and outcomes in patients diagnosed with gliosarcoma.

\section{References}

1. Kozak KR, Mahadevan A, Moody JS. Adult gliosarcoma: epidemiology, natural history, and factors associated with outcome. Neuro Oncol. 2009;11(2):183191.

2. di Norcia V, Piccirilli M, Giangaspero F, Salvati M. Gliosarcomas in the elderly: analysis of 7 cases and clinicopathological remarks. Tumori. 2008;94(4):493-496

3. Louis DN, Ohgaki H, Wiestler OD, Cavenee WK, Burger PC, Jouvet A, Scheithauer BW, Kleihues P: The 2007 WHO classification of tumours of the central nervous system. Acta Neuropathol. 2007;114: 97-109

4. Lutterbach J, Guttenberger R, Pagenstecher A. Gliosarcoma: a clinical study. Radiother Oncol. 2001;61(1):5764.

5. Stroebe H. Uber entstehung und bau der hirngliome. BeitrPatholAnat1895;18:40586

6. Biswas A, Kumar N, Kumar P, Vasishta RK, Gupta K, Sharma SC, Patel F. et al. Primary gliosarcoma - clinical experience from a regional cancer centre in north India. Br J Neurosurg. 2011;25(6):723729

7. Kumar P, Singh S, Krishnani N, Datta NR. Gliosarcoma: an audit from a single institution in India of 24 post-irradiated cases over 15 years. J Cancer Res Ther. 2008;4(4):164-168.

8. Kakkar N, Kaur J, Singh GK, Singh P, Siraj F, Gupta A. Gliosarcoma in Young Adults: A Rare Variant of 
Glioblastoma. World J Oncol. 2017;8(2):53-57.

9. Witwer BP, Salamat MS, Resnick DK: Gliosarcoma metastatic to the cervical spinal cord: case report and review of the literature. Surg Neurol. 2000;54: 373-378

10. Vukelic Z, Kalanj-Bognar S, Froesch M, Bindila L, Radic B, Allen M, PeterKatalinic J. et al. Human gliosarcomaassociated ganglioside composition is complex and distinctive as evidenced by high-performance mass spectrometric determination and structural characterization. Glycobiology. 2007;17( 5):504-515.

11. Galanis E, Buckner JC, Dinapoli RP, Scheithauer BW, Jenkins RB, Wang CH, O'Fallon JR. et al. Clinical outcome of gliosarcoma compared with glioblastoma multiforme: North Central Cancer Treatment Group results. J Neurosurg. 1998;89(3):425-430.

12. Khanna M, Siraj F, Chopra P, Bhalla S, Roy S. Gliosarcoma with prominent smooth muscle component (gliomyosarcoma): a report of 10 cases Indian J Pathol Microbiol. 2011;54(1):51-54.

13. Salvati M, Caroli E, Raco A, Giangaspero F, Delfini R, Ferrante L. Gliosarcomas: analysis of 11 cases do two subtypes exist? J Neurooncol. 2005;74(1):59-63

14. Faried, A., Hernowo, B.S., Adam, A., Arifin, M.Z. Temporoparietal Gliosarcoma: A Case Report with Literature Review. Indonesian Journal of Neurosurgery 2018; 1(1): 19-22

15. Beaumont TL, Kupsky WJ, Barger GR, Sloan AE: Gliosarcoma with multiple extra-cranial metastases: case report and review of literature. $\mathrm{J}$ Neurooncol. 2007;83: 39-46

16. Han SJ, Yang I, Tihan T, Prados MD, Parsa AT. Primary gliosarcoma: key clinical and pathologic distinctions from glioblastoma with implications as a unique oncologic entity. J Neurooncol. 2010;96(3):313-320.

17. Walker GV, Gilbert MR, Prabhu SS, Brown PD, McAleer MF. Temozolomide use in adult patients with gliosarcoma: an evolving clinical practice. J Neurooncol. 2013;112(1):83-89

18. Han SJ, Yang I, Tihan T, Chang SM, Parsa AT. Secondary gliosarcoma: a review of clinical features and pathological diagnosis. J Neurosurg. 2010;112(1):26-32. 